\title{
TPEN, zinc chelator, inhibits biofilm and hyphae formation by Trichosporon asahii
}

P429

OSanae Kurakado, Takashi Sugita

Department of microbiology, Meiji pharmaceutical university, Tokyo, Japan

\section{Background}

Trichosporon species are the second most common etiological agent in catheter-related fungemia, followed by Candida species. However, the clinical outcome of disseminated trichosporonosis is poorer than that of disseminated candidiasis, and the mortality rate is $>70 \%$. Trichosporon asahii is the major causative agent of both disseminated and deep-seated trichosporonosis. This fungus exists both as a yeast and as hyphae and arthroconidia. The assimilation of essential nutrients is critical to the ability of $T$. asahii to proliferate, and to form and maintain biofilms (BFs), an important cause of fungemia. To identify the role played by metal cations in the process, we evaluated the effects of cation chelators on BF formation by $T$. asahii, and on the morphological versatility of this fungus.

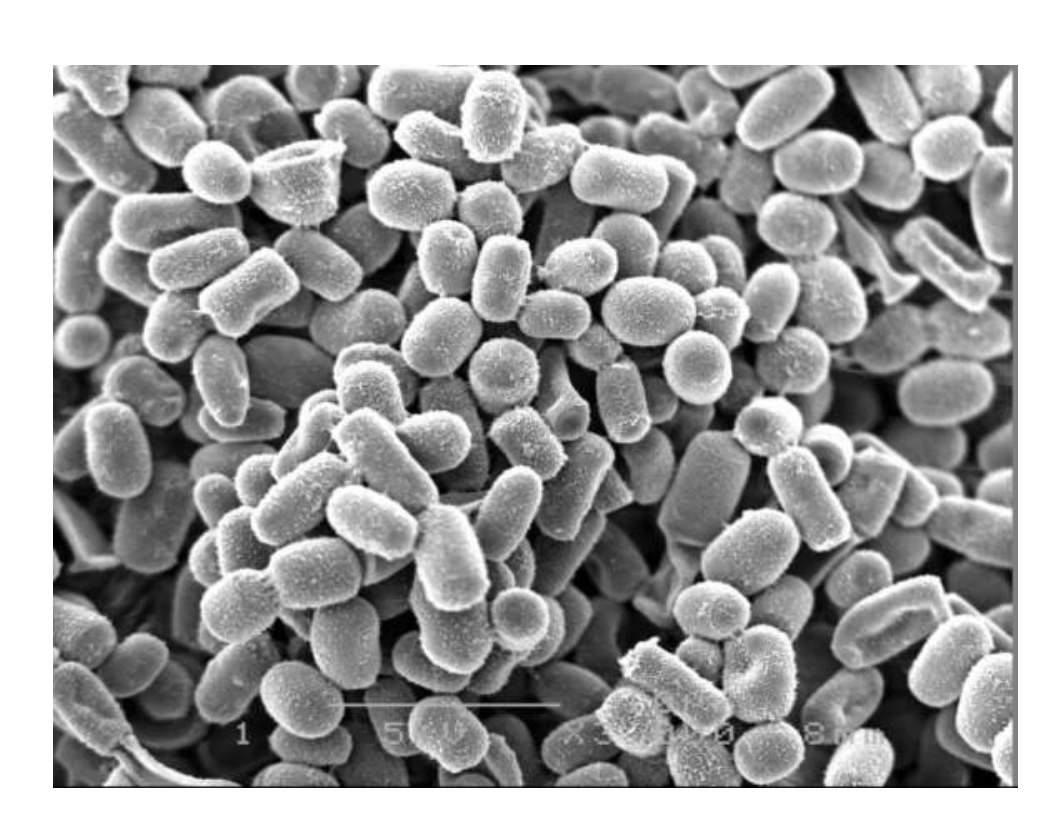

Scanning electron micrograph of T. asahii

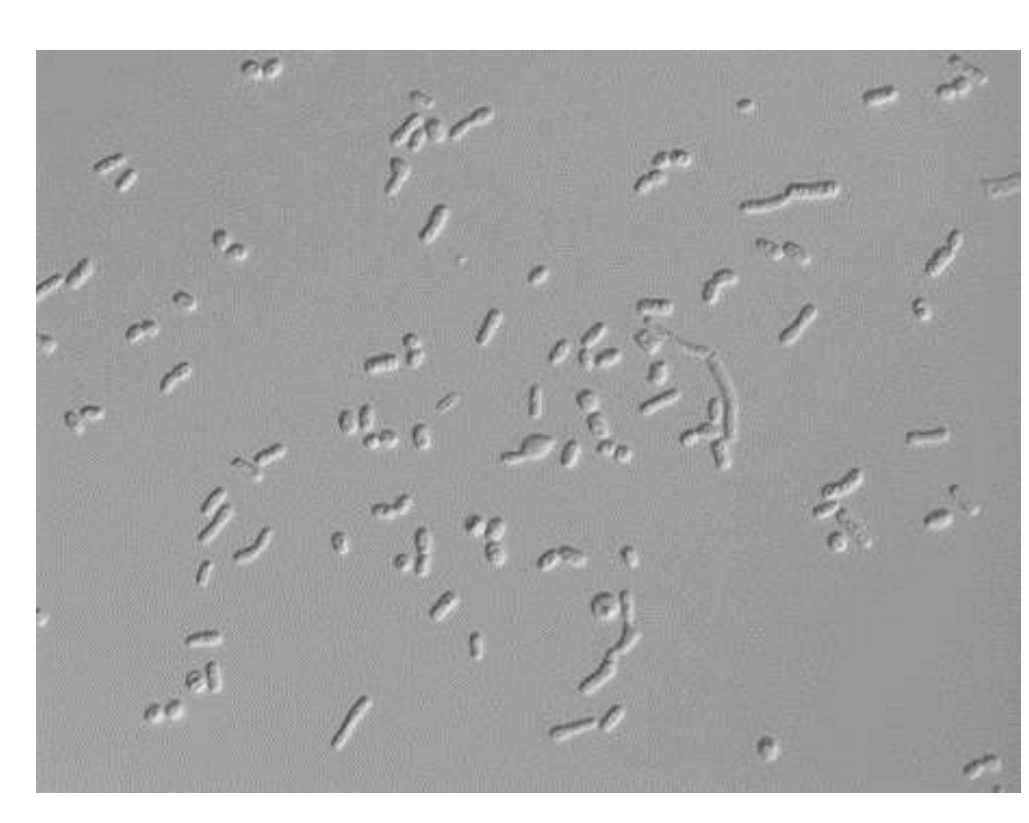

Yeast form of $T$. asahi

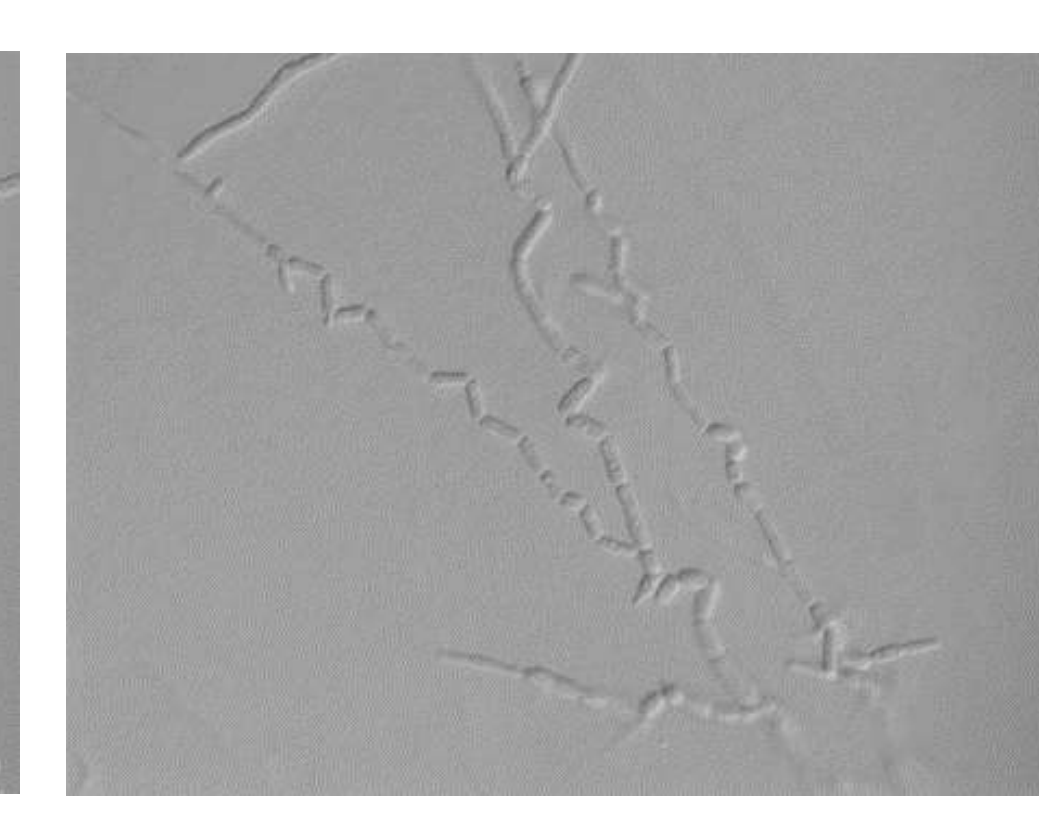

Arthroconidia form of $T$ asahii
Anti-biofilm effect by chelators

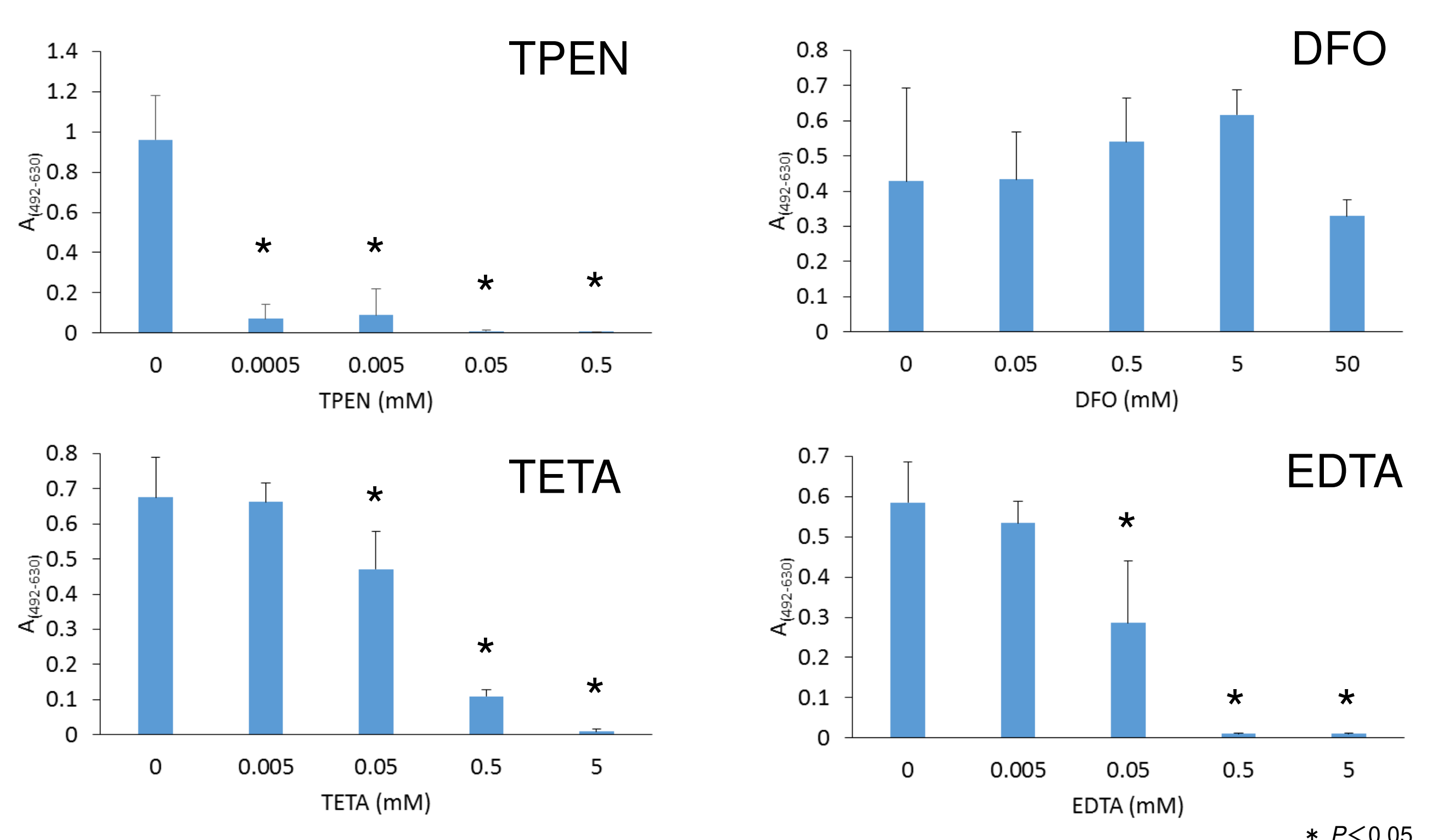

TPEN, N,N,N;'N-Tetrakis(2-pyridylmethyl)ethylenediamine; DFO, Deferoxamine; TETA, Triethylenetetramine

T TPEN (zinc chelator) reduced BF formation more efficiently than did other chelators.

\section{Effect of zinc on biofilm formation}

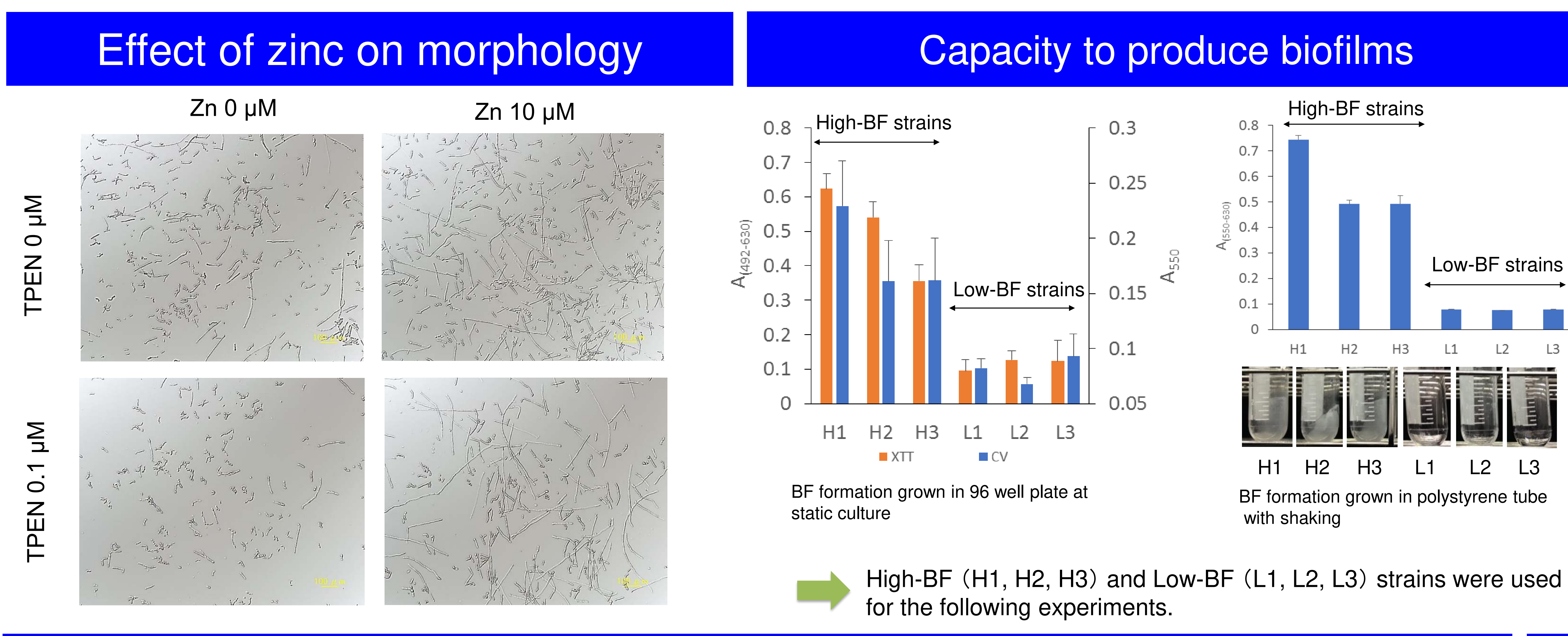

Relationship between the capacity to form biofilms and morphology
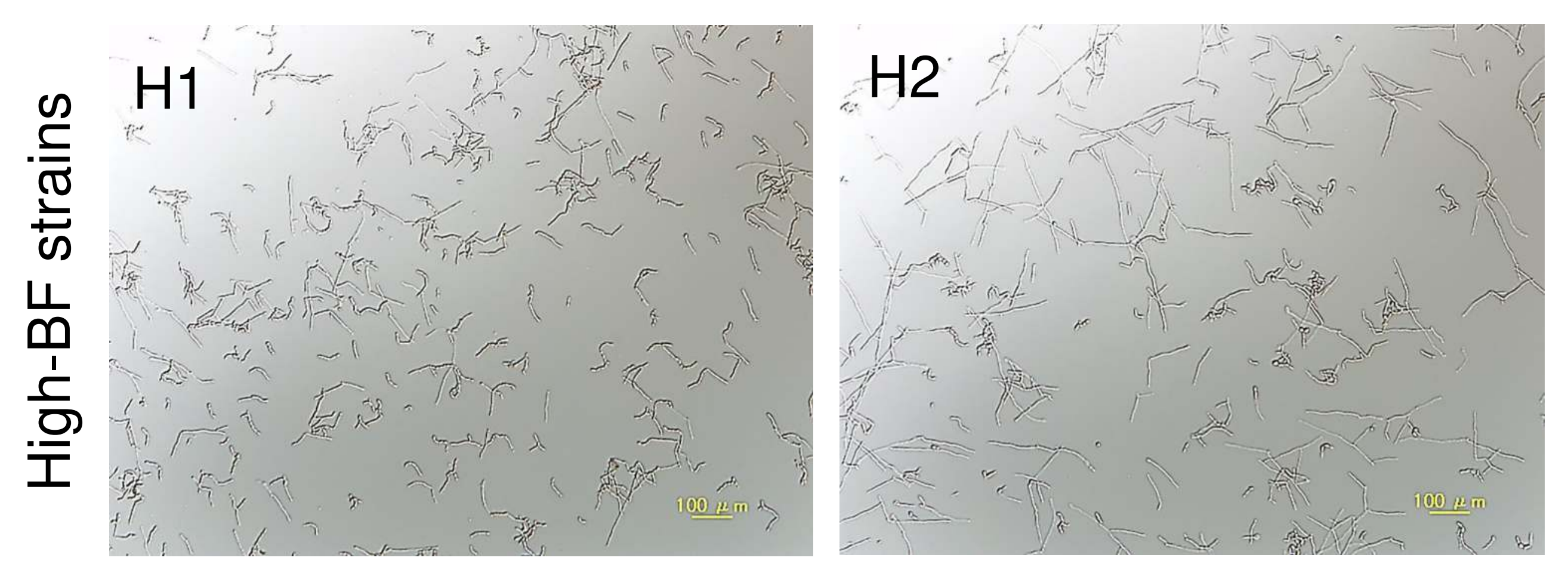

里

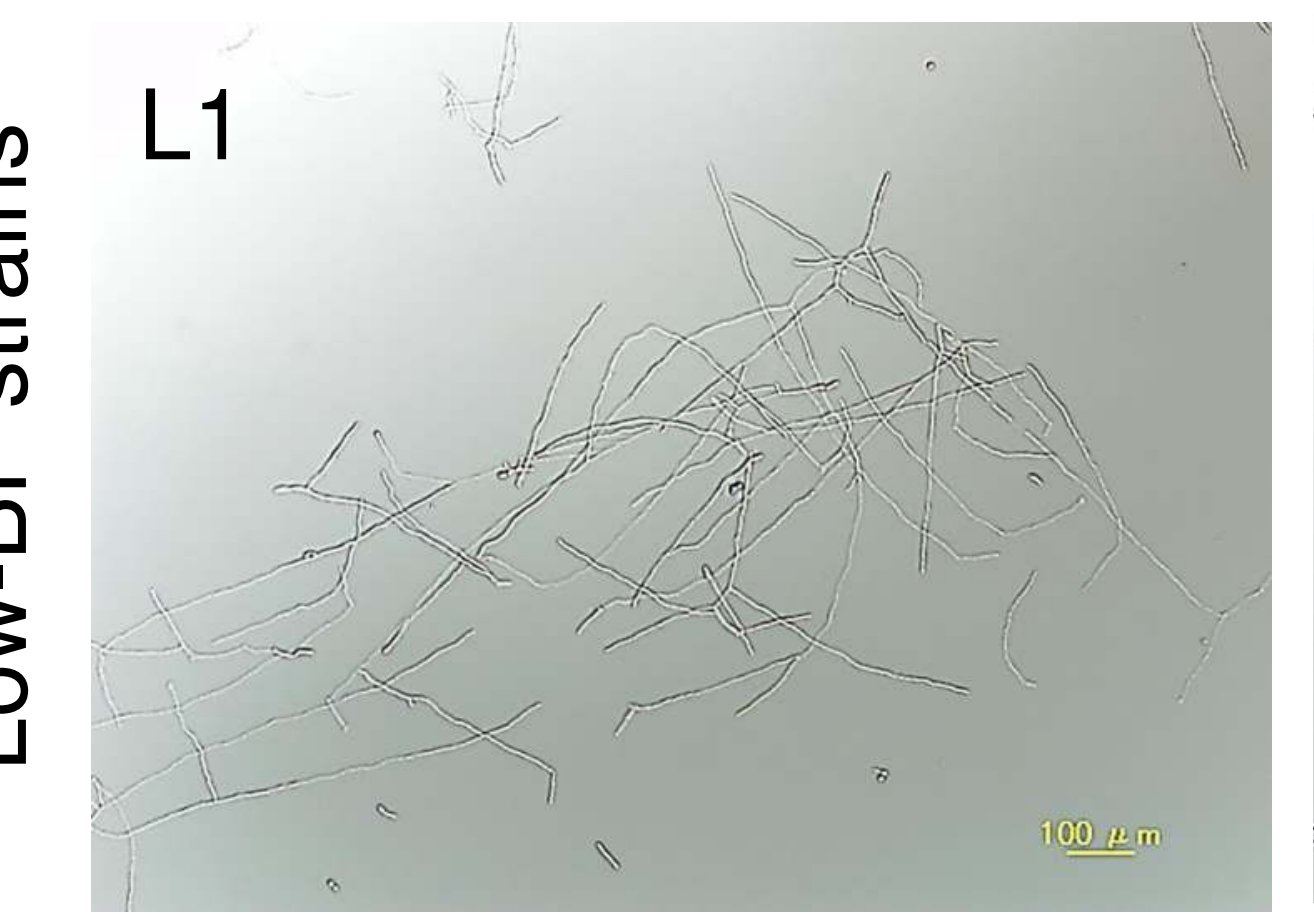

H3

L2

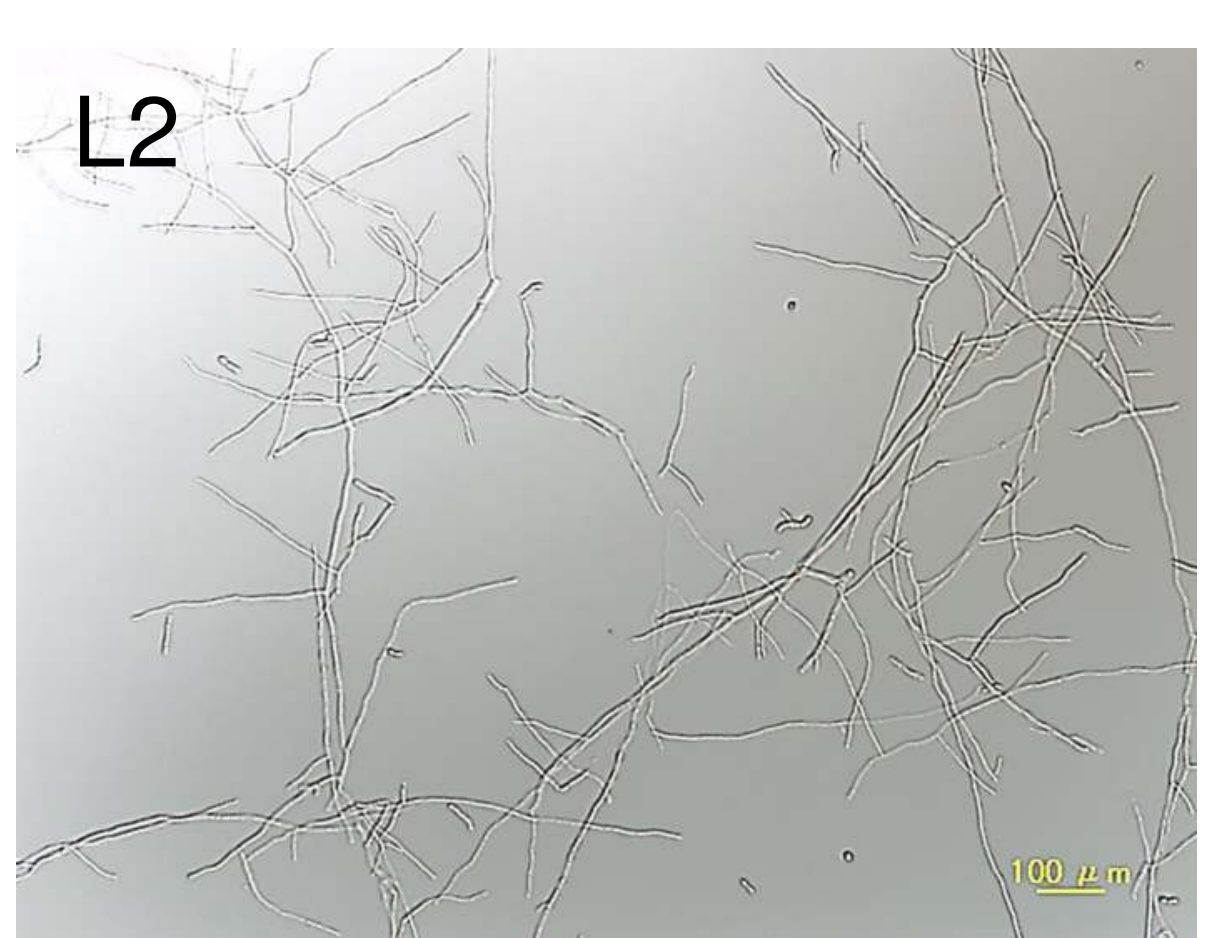

L3
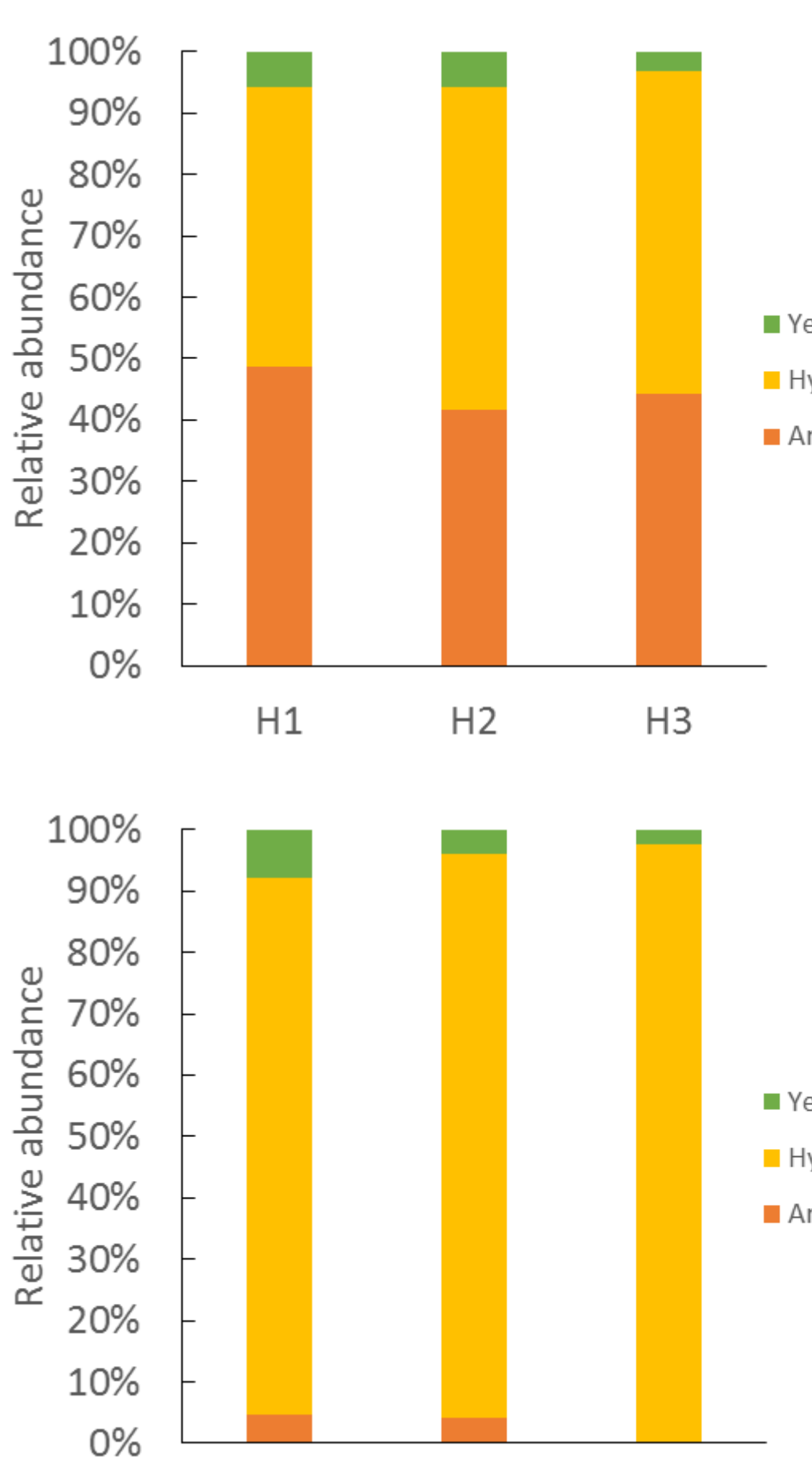

Incubate with TPEN and Zn
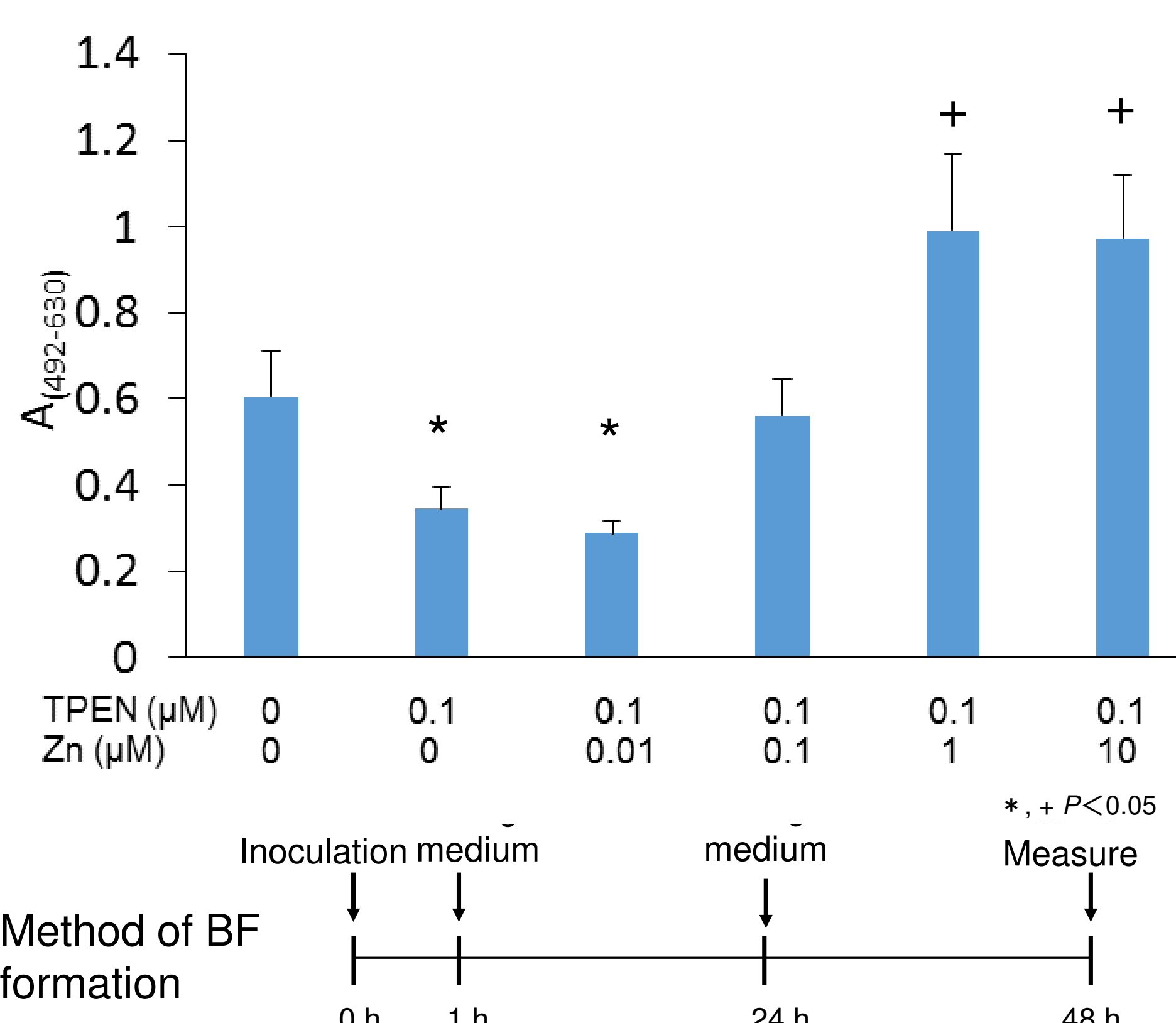

Incubate with TPEN $\rightarrow$ Change medium containing Zn

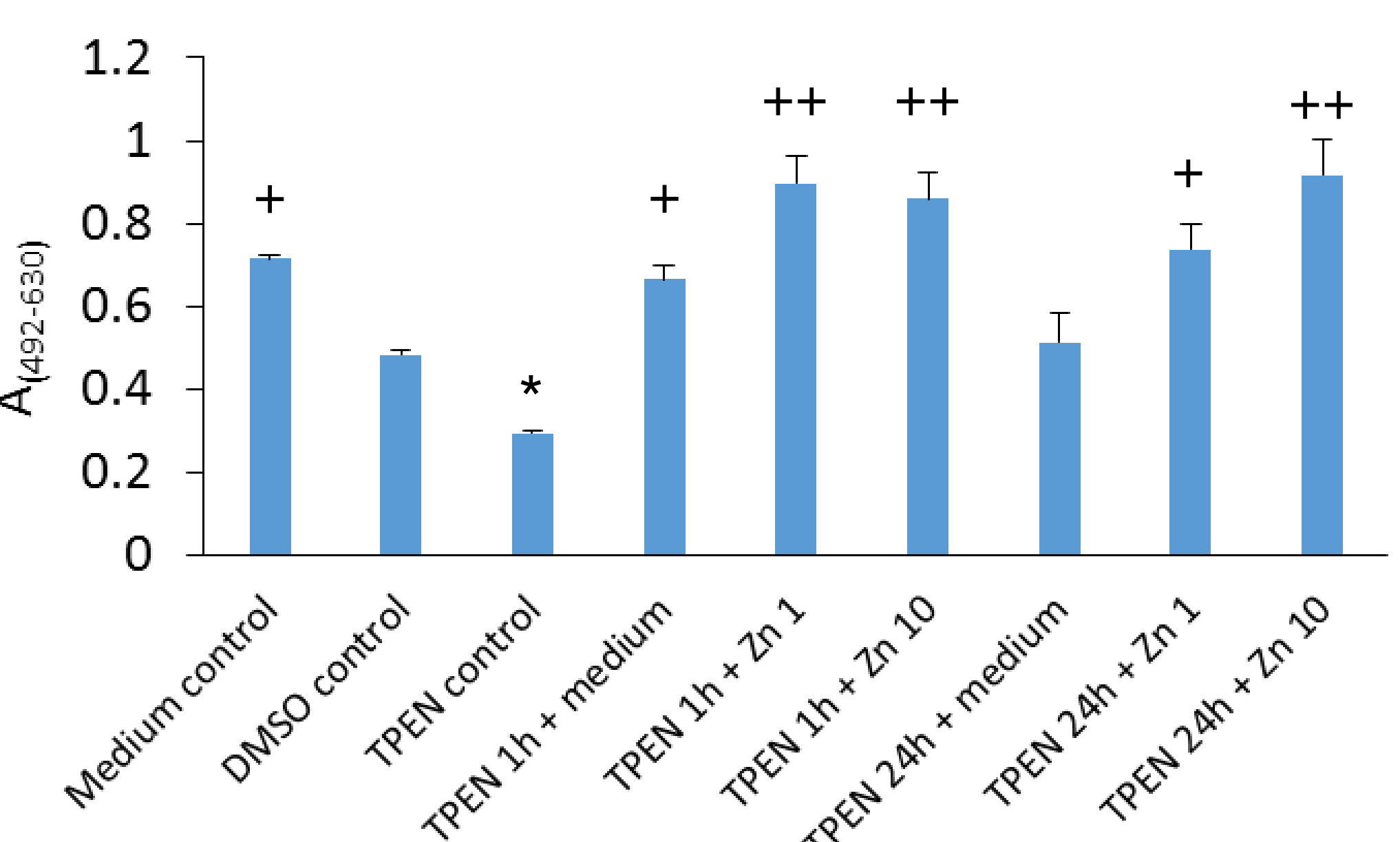

Adding zinc not only recovered, but also promoted BF formation.
Cell surface hydrophobicity

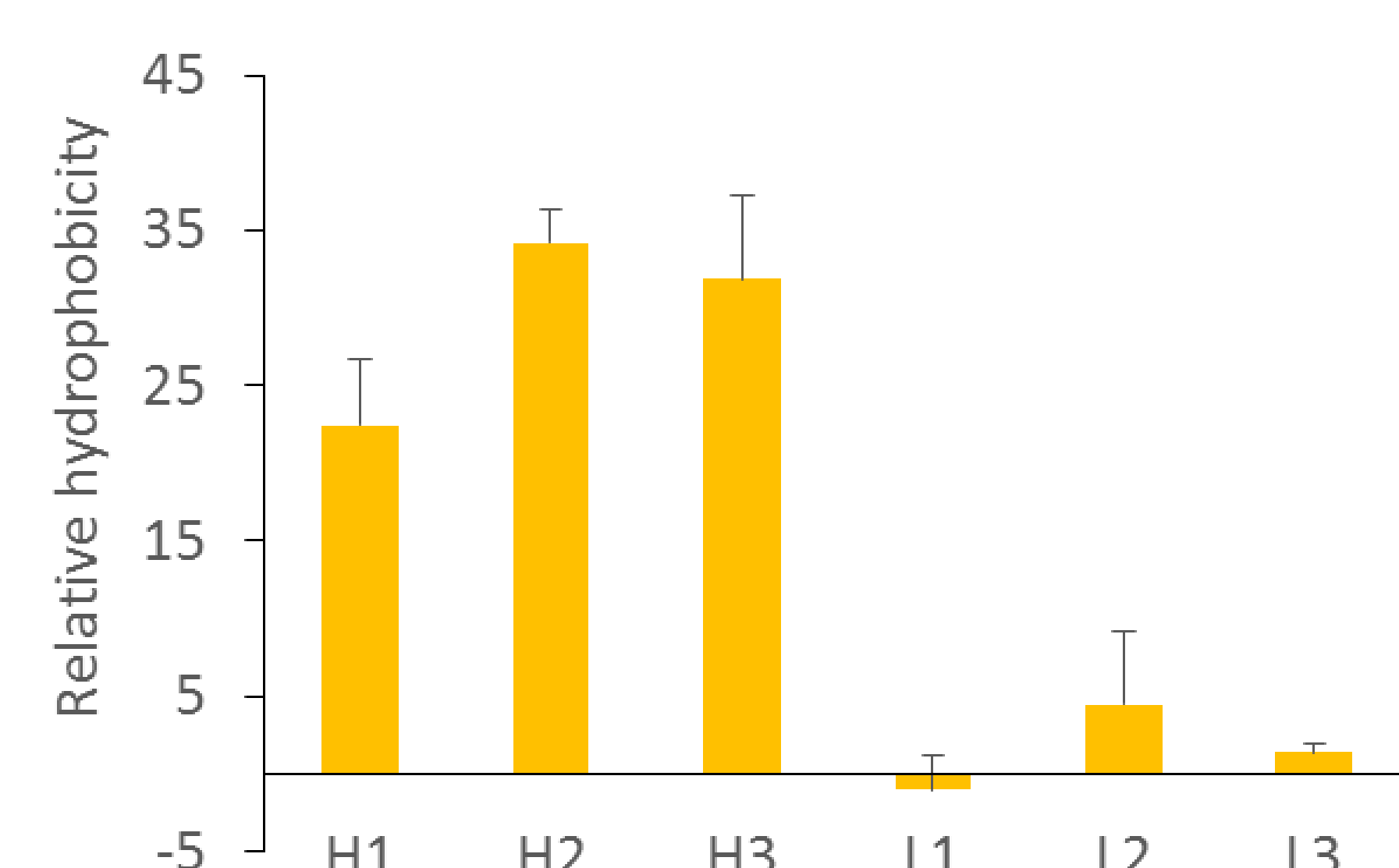

The cell surface hydrophobicity of the High-BF strains exceeded that of the Low-BF strains.

\section{cosere}

Adherence

The adherence of arthroconidia and maturation by hyphal elongation may be critical to $\mathrm{BF}$ formation by $T$. asahii.

High-BF strains produced abundant arthroconidia, while Low-BF strains mainly produced hyphae, with a few arthroconidia.

\section{Results and Discussion}

- Cation chelation using TPEN (zinc chelator) reduced BF formation more efficiently than did cation chelation using other chelators.

Adding zinc not only recovered, but also promoted BF formation

Zinc promoted hyphal elongation.

- High-BF strains of $T$. asahii produced abundant arthroconidia, while Low-BF strains produced mainly hyphae, with a few arthroconidia.

- The cell-surface hydrophobicity of the High-BF strains exceeded that of the Low-BF strains. 